\title{
Barreras de regulación para la inserción de las energías renovables en sistemas de generación distribuida a pequeña escala, caso Nicaragua
}

\section{Regulation barriers for the insertion of renewable energies in small-scale distributed generation systems, Nicaragua case of study}

\author{
Napoleón V. Blanco Orozco \\ Universidad Nacional de Ingeniería. Facultad de Electrotecnia y Computación, Departamento de Ingeniera \\ Eléctrica. Managua, Nicaragua \\ napoleon.blanco@fec.uni.edu.ni;blanconapoleon@yahoo.com
}

(recibido/received: 17-octubre-2021; aceptado/accepted: 30-noviembre-2021)

\section{RESUMEN}

En este artículo se detectaron y analizaron las diferentes barreras que se presentan como un reto a mejorar para la inserción de las Energías Renovables (ER) en sistemas de generación distribuida a pequeña escala utilizando la teoría fundamentada y la herramienta informática Atlas ti 7. Se encontró que se debe mejorar el marco normativo para hacer atractiva la inversión privada en Energías Renovables (ER) conectadas en sistemas de generación distribuida y se lograron identificar los diferentes tipos de barreras al empleo de las energías renovables a las redes eléctricas de las compañías distribuidoras de energía en base al estudio del caso nicaragüense.

Palabras claves: Energías Renovables, barreras, generación distribuida.

\begin{abstract}
In this article, different barriers that are presented as a challenge to improve for the insertion of Renewable Energies (RE) in small-scale distributed generation systems were detected and analyzed using the grounded theory and the computer tool Atlas ti 7. It was found that the regulatory framework should be improved to make private investment in Renewable Energies (RE) in distributed generation systems attractive and it was possible to identify different types of barriers to the use of renewable energies in the electrical networks of power distribution companies based on to the Nicaraguan case study.
\end{abstract}

Keywords: Renewable energy, barriers, distributed generation.

\section{INTRODUCCIÓN}

Nicaragua forma parte de un esfuerzo regional para establecer un mercado eléctrico que incluye a centroamericana, Panamá y México para que el sector eléctrico sea competitivo y que abastezca en forma oportuna y sostenible la electricidad requerida para el desarrollo económico y social. Así mismo, para formar condiciones técnicas para la integración energética de nuestros países se deben de realizar esfuerzos para lograr que en el sector energético participen tanto empresas de capital público como empresas con 
participación privada con el fin de desarrollar, diseñar, financiar, construir y mantener en funcionamiento los sistemas eléctricos.

Análogamente con el fin de establecer de asegurar el suministro energético futuro, mejorar la disponibilidad y la regulación de la energía eléctrica, se debe de proveer de energía más barata, limpia y de calidad a la población; por lo tanto, es necesario el fomento de las energías renovables en la matriz de generación de cada uno de nuestros países latinoamericanos.

Por otra parte, la evolución histórica de la capacidad de generación de energía eléctrica ha sufrido crisis como la que vivió el Sistema Eléctrico en los años 1995-1997, llegándose inclusive a racionar el suministro; esta crisis se comenzó a solucionar con la entrada de nuevas plantas, desgraciadamente todas ellas térmicas, a partir de contrataciones con empresas privadas las cuales contaron con contratos de potencia (PPA Power Purchase agreement), realizadas en esos años, lo que le permitió recuperar rápidamente la inversión y encareció el precio final de la energía por los precios de potencia altos en que fueron negociados por funcionarios del estado de entonces. Actualmente, la potencia instalada es 1,317 MW de la cual el 54\% corresponde a plantas de generación que utilizan derivados del petróleo (INE, 2021), lo cual no indica que a pesar de los esfuerzos por independizar la matriz energética del petróleo aún hay mucho por hacer.

Nicaragua cuenta con recursos energéticos renovables y con reservas explotables considerables lo que se necesita es atraer inversión para asegurar el suministro y para atraer la inversión en energías renovables por lo que se debe de promover el empleo de estos energético y una forma de hacerlo es de librar la inserción de estas fuentes energéticas de obstáculo o barreras que no favorezcan su emprendimiento. Aunque es posible encontrar muchos beneficios de las energías renovables incluyendo la protección del medio ambiente y las des carbonización de la economía, se puede observar que existe un aumento de las inversiones en Energías Renovables (ER) de gran escala en países desarrollados, pero en los en vías de desarrollo existe un lento desarrollo de programas de ER. No obstante, existen un gran número de barreras que impiden la inserción de las ER en forma de generación distribuida que se caracteriza por la generación de energía eléctrica en los lugares próximos a las cargas de forma descentralizada.

En consecuencia, el propósito de este artículo es analizar las diferentes barreras que se presentan como un reto a mejorar para la inserción de las Energías Renovables (ER) en sistemas de generación distribuida a pequeña escala y para este propósito se ha procedidos a revisar el marco normativo de Nicaragua con respecto a las energías renovables y se han identificado los tipos de barreras para el empleo de estas fuentes energéticas.

\section{METODOLOGÍA}

El proceso Este estudio fue de tipo explicativo y con enfoque cualitativo con el propósito de analizar y luego sintetizas las barreras para la inserción de las energías renovables en sistemas de generación distribuida. Inicialmente, se realizó una revisión de la perspectiva teórica desarrollando una vertebración de las citas bibliográficas con el propósito de ordenar la extracción de la información usando la teoría fundamentada. De la misma forma, los referentes teóricos fueron caracterizados utilizando la herramienta informática Atlas ti 7 para el desarrollo del análisis documental.

Ahora bien, se realizó un análisis de los documentos infiriendo los elementos que impiden el empleo de las ER para la generación de energía eléctrica en sistemas distribuidos con un proceso de construcción y análisis e integración. Sucede pues, que el proceso de sustentación incluyó el análisis de citas, códigos, memos, familias de códigos, y las relaciones entre estos elementos a través del examen de redes hasta llegar a la saturación teórica. 
Además, se empleó el método analítico para la extracción de los diferentes tipos de barrera a la inserción de ER en las redes eléctricas con el objeto de estudiarlas y examinarlas por separado, para ver, por ejemplo, las relaciones entre éstas y encontrar posibles relaciones que existe entre los obstáculos encontrados. Así mismo, se empleó el método comparativo entre los factores en estudio de las barreras a la ER con fines de generalización y de verificación relaciones. Finalmente, se empleó el método de síntesis mediante el cual se relacionaron los diferentes tipos de barreras a la inserción de las energías renovables al sistema eléctrico de Nicaragua.

\section{RESULTADOS}

En la tabla 1 se procede a analizar las principales leyes y normativas que se relacionan al empleo de las energías renovables y su inserción en las redes eléctricas del sistema Interconectado Nacional de Nicaragua (SIN).

Tabla 1. Análisis del marco normativo nicaragüense y su relación con las barreras a la inserción de las energías renovables al SIN.

\begin{tabular}{|c|c|c|}
\hline Marco legal & Descripción & $\begin{array}{c}\text { Relación Con las } \\
\text { Energias Renovables }\end{array}$ \\
\hline Ley de Industria Eléctrica & $\begin{array}{l}\text { Esta ley establece el régimen legal } \\
\text { sobre las actividades de la industria } \\
\text { eléctrica; generación, } \\
\text { transmisión, } \\
\text { comercialización, importación y } \\
\text { exportación de la } \\
\text { energía eléctrica. }\end{array}$ & $\begin{array}{l}\text { Se instituye que todo agente } \\
\text { económico pude conectarse al SIN } \\
\text { cumpliendo con las normas } \\
\text { técnicas establecidas indicadas en } \\
\text { el reglamento de operación. } \\
\text { Además, los agentes económicos } \\
\text { propietarios de líneas y demás } \\
\text { elementos del } \\
\text { sistema de transmisión están } \\
\text { obligados a permitir la conexión a } \\
\text { sus } \\
\text { instalaciones, a los demás agentes } \\
\text { económicos, previo cumplimiento } \\
\text { de las normas que rigen el servicio } \\
\text { y el pago de las retribuciones que } \\
\text { correspondan. }\end{array}$ \\
\hline $\begin{array}{l}\text { Reglamento de la ley de industria } \\
\text { Eléctrica, decreto } 42.98\end{array}$ & $\begin{array}{l}\text { Este reglamento regula la ley de } \\
\text { Industria Eléctrica Ley } 272 \text {. El MEM } \\
\text { es la institución encargada de velar } \\
\text { por la aplicación de la ley. }\end{array}$ & $\begin{array}{l}\text { Se especifica que un agente } \\
\text { económico puede generar, } \\
\text { transmitir y distribuir solo en el } \\
\text { caso de sistemas aislados. Y de } \\
\text { estar conectado al SIN puede } \\
\text { distribuir para una capacidad de } \\
\text { generación menor o igual a } 10,000 \\
\text { KW. }\end{array}$ \\
\hline & & $\begin{array}{l}\text { Ciertamente, para generar debe ser } \\
\text { un agente económico con licencia } \\
\text { de generación. }\end{array}$ \\
\hline $\begin{array}{l}\text { Reglamento de la ley de exploración y } \\
\text { explotación de } \\
\text { recursos geotérmicos. Decreto no. } 45 \text { - } \\
2010 .\end{array}$ & $\begin{array}{l}\text { El Reglamento tiene por objeto } \\
\text { reglamentar y regular las } \\
\text { disposiciones de la Ley No. } 443 \text {, "Ley } \\
\text { de Exploración y Explotación de } \\
\text { Recursos Geotérmicos". }\end{array}$ & $\begin{array}{l}\text { El marco normativo favorece a la } \\
\text { inversión y el empleo de la energía } \\
\text { geotérmica. }\end{array}$ \\
\hline Normativa de operación & $\begin{array}{l}\text { El propósito de esta normativa es fijar } \\
\text { las reglas de carácter operativo del } \\
\text { Sistema Interconectado Nacional } \\
\text { (SIN) y el Sistema }\end{array}$ & $\begin{array}{l}\text { En esta ley se clasifica como } \\
\text { generador no despachable aquel } \\
\text { que no cuenta con potencia firma } \\
\text { contante despachable, por ejemplo: } \\
\text { plantas hidráulicas a filo }\end{array}$ \\
\hline
\end{tabular}




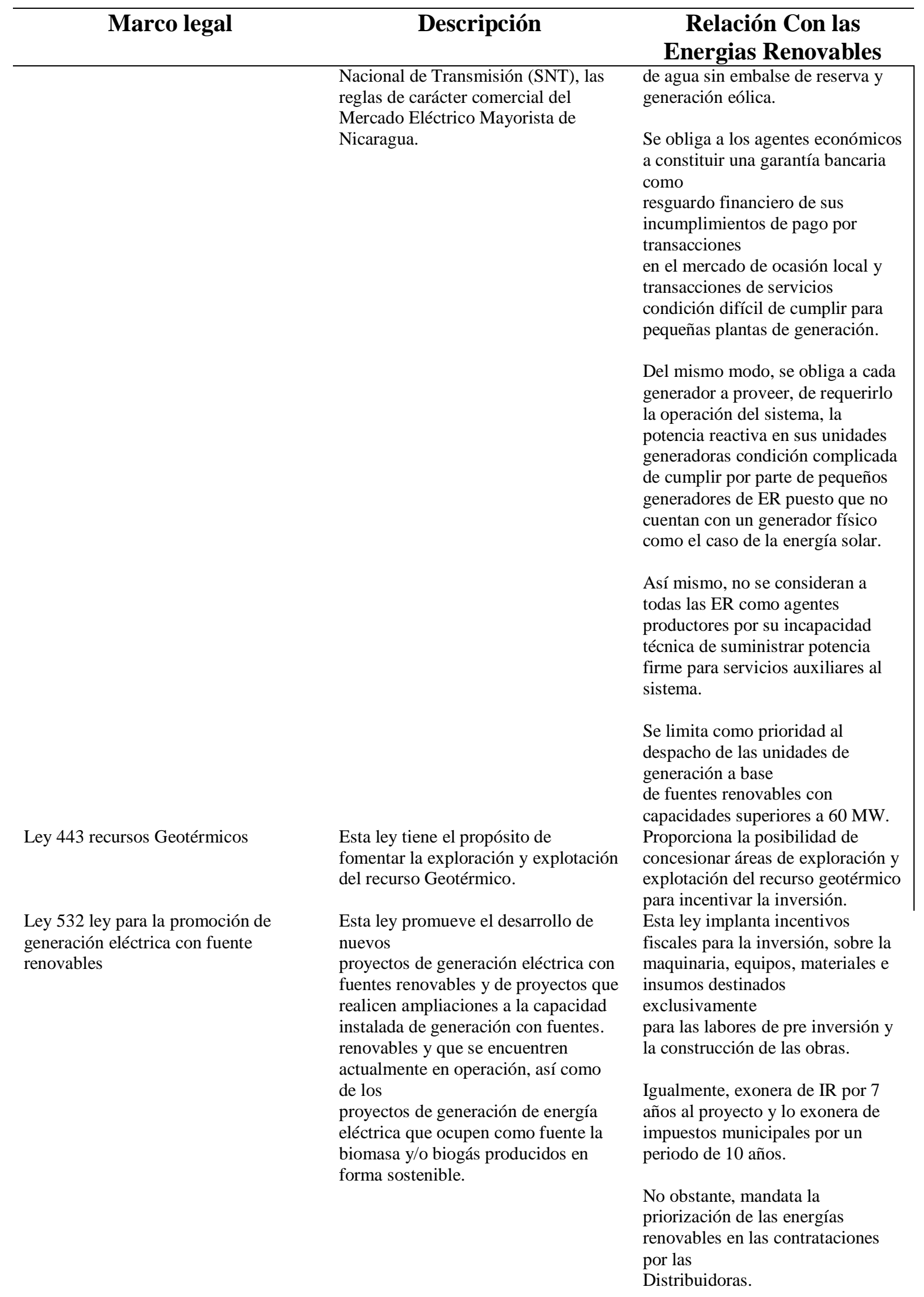


Mientras que como factor de barrera se tiene que se establece una banda de precios deberán vender esta energía en el mercado de ocasión interno de acuerdo a sus precios promedios diarios, manteniéndose dentro de una banda de precios no menores de 5.5 centavos de dólar por KW. ni mayor de 6.5 centavos de dólar por Kwh.

Impone requerimientos técnicos como el suministrito de reserva rodante y otros servicios auxiliares que en su mayoría no pueden ser cumplidos por las fuentes de Energias Renovables.

Se incluyen estudios especiales para la inserción delas energías renovables como el caso de la energía eólica que con el fin de ser habilitada para conectarse al SIN, los desarrolladores de cualquier proyecto eólico deberán realizar estudios necesarios a fin de demostrar que dicha generación no

Acuerdo ministerial sobre generación distribuida.

Resolución ministerial sobre banda de precios de las Energias Renovables
Establece requisitos y procedimientos y responsabilidades administrativas y comerciales para la generación con ER para autoconsumo a conectarse en sistemas de distribución.

Establecer una banda de precios de energía de referencia. causa disturbios a la operación del SIN.

Esta ley regula la generación de energía eléctrica con energías renovables para autoconsumo menor a $5 \mathrm{MW}$

Al generador conectado bajo esta ley no se le considera agente económico por lo que no puede intervenir en el mercado eléctrico

Indica que la conexión es en las instalaciones internas del agente.

El generador debe asumir costos de inspección de la empresa distribuidora y asumir los daños que pudiera ocasionar a la estabilidad de la red.

La remuneración por excedente solo es posible cuando se haya firmado un contrato con la distribuidora y será de un valor máximo al $80 \%$ del precio más bajo de la banda de precios para las ER

La banda de precios de las energías renovables este entre 


\begin{tabular}{|c|c|c|}
\hline Marco legal & Descripción & $\begin{array}{c}\text { Relación Con las } \\
\text { Energias Renovables }\end{array}$ \\
\hline & & $\begin{array}{l}\text { US\$/MW-HR } 70 \text { para la energía } \\
\text { solar hasta un máximo de } \\
\text { US\$/MW-HR } 107 \text { para la } \\
\text { hidroeléctrica a filo de agua, } \\
\text { cuando el precio de las plantas } \\
\text { térmicas está por encima del } \\
\text { máximo de esta banda. }\end{array}$ \\
\hline
\end{tabular}

Por otra parte, al realizar el análisis documental utilizando la teoría fundamentada y con el soporte informático del atlas ti 7 se obtuvieron las relaciones entre códigos relacionados en forma de causa efecto con respecto a la temática investigada (ver resultados de la figura 1) y se encontró que el marco legal que compete a las energías renovables, aunque su propósito es incentivar la inversión en estas fuentes en algunos aspectos se convierte en una barrera de inserción. Así, se encontró que hay aspectos económicos como las tasas de interés aplicadas al financiamiento de estas inversiones y los incentivos tributarios y fiscales no son suficientemente atractivos en comparación con los obtenidos por la tecnología basada en petróleo; aun peor en la legislación nicaragüense se dictaminó una banda de precios de venta de la energía renovable que está por debajo de los precios reconocidos a las otras plantas generadoras, lo que amerita un análisis económico más profundo.

Por otra parte, se detectan barreras de tipo técnicas en el marco normativo como la dificultad en reconocer a un generador con energía renovable como agente del mercado eléctrico por su limitante técnica de no poseer potencia firme y que solo está destinado al suministro de energía eléctrica a través de un balance de generación y consumo con un reconocimiento de la diferencia en la factura del servicio.

Del mismo modo, se encontró que es menester mejorar las políticas energéticas que favorezcan a las ER y que inician por la superación de barreras culturales hacia un cambio del modelo energético hacia la eficiencia energética y ahorro de energía que disminuya el consumo y así poder hacer viable y factible la sustitución en gran parte de la energía basada en combustible fósil.

Además, del análisis de citas, códigos y las relaciones entre estos se determinó que el modelo energético por adoptar estaría sustentado en las energías renovables toda vez que estas son fuente autóctona, con pocos impactos ambientales y por tanto con una reducción de las externalidades negativas de la producción energética al medio ambiente lo que se observa en la figura 1.

Con respecto a las barreras a la inserción de las ER a las redes eléctricas se puede decir de manera general que surgen de la resistencia al cambio de un modelo energético actual sustentado en el consumo de derivados del petróleo que conlleva toda una industria integrada verticalmente y que resiste su sustitución por un nuevo modelo energético sostenible que incluya el empleo de las energías renovables y la eficiencia energética. En este sentido, Elgamal (2020) destaca que si se combinan políticas públicas, subsidios y financiamiento, fue fundamental para el desarrollo de la energía solar en algunos países de su estudio; no obstante, en el caso de Brasil refiere que las políticas públicas están fragmentadas e incompletas y que a pesar de existir mecanismos de financiamiento y bajas tasas de interés a largo plazo para los fabricantes, inversionistas y consumidores estas medidas han sido insuficientes para para aumentar el empleo de la energía solar.

Por otra parte, Ortiz et al. (2012) señalan que a pesar de las ventajas del empleo de las ER tienen como su menor impacto ambiental existe muy poca voluntad política para incentivar la inversión y promover el desarrollo de proyectos ER y que de manera contraproducente en algunos países el marco normativo en vez de presentarse como una herramienta que suscite la implementación de las ER, se torna en un efecto negativo para la inversión privada y refiere como ejemplo de políticas contraproducentes los subsidios a los combustibles; Lara (2015) propone una política de subvenciones a las ER. Por su parte, Turiel (2012) remarca la disminución de disponibilidad de recursos energéticos 
y, por tanto, con el modelo energético actual las ER son inviables dado que estas no podrían sustituir a los combustibles fósiles; lo anterior tiene sentido dado que las ER deben ser acompañadas por un modelo energético sostenible que busque la eficiencia energética y la reducción del consumo de energéticos y su aprovechamiento sustentable.

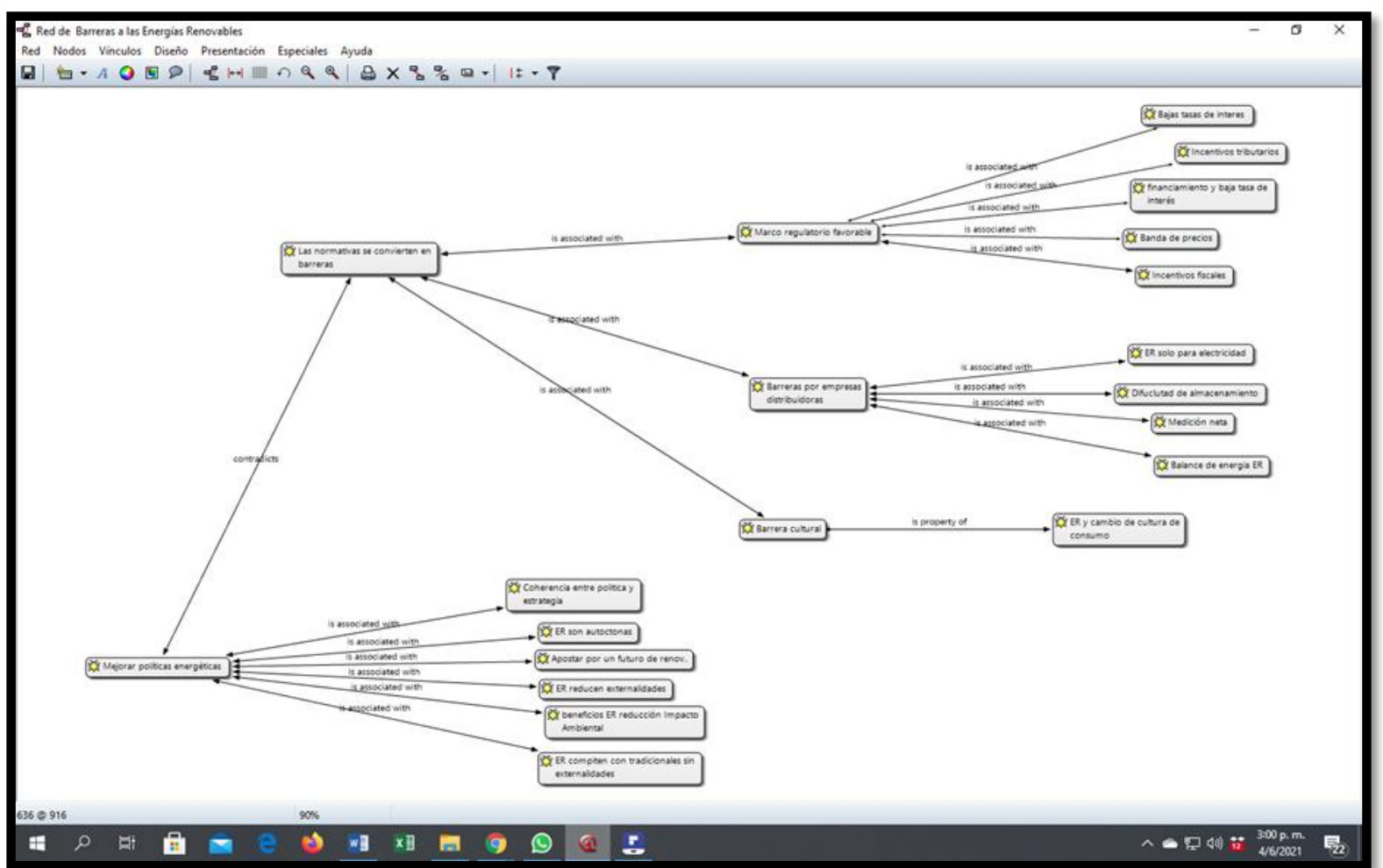

Figura 1. Resultado del análisis de red de códigos relacionados a las barreras de inserción de las energías renovables a los sistemas eléctricos de potencia.

De manera análoga, Sanabria et al (2014) expresan que el modelo de desarrollo más aplicado es de tipo centralizado de arriba hacia abajo; impuesto desde intereses externos y luego, a nivel nacional se derivan macro programas diseñados desde los cuales se determina la planificación de suministros energéticos. Por lo que, las fuentes de energías renovables, al utilizar menos servicios y productos importados y al utilizar principalmente los recursos locales, contribuyen al progreso de la industria eléctrica a nivel local. En este mismo, sentido, Luna y Ramírez (2018) recuentan que México tiene un marco legal favorable a las ER y como ejemplo expresan que, el consumidor puede tener pequeños sistemas fotovoltaicos que les permitirá un ahorro en el pago de energía eléctrica; además de que puede obtener un ingreso por la venta de energía a la Comisión Federal de Electricidad.

Del mismo modo, López et al (2014) resaltan que existe una preocupación mundial por los temas ambientales y el aseguramiento energético de los países. Y que estas dos problemáticas han favorecido que los gobiernos y entes privados analicen las posibilidades de la implementación de diferentes modelos de sistemas eléctricos. Por su parte, Cordech (2009) afirma que las energías renovables y las políticas de reducción de demanda no serán suficientes para garantizar la satisfacción de las necesidades energéticas futuras; este autor opina que apostar por un futuro de renovables aumentaría los costes energéticos y empeoraría nuestra competitividad internacional y por el contrario se deben de potenciar las diferentes energías renovables a sabiendas de que algunos opinan que el sistema actual centralizado no las puede insertar y propone que las redes de distribución deben de adaptarse a las nuevas formas de generación distribuida.

Por lo consiguiente se pueden distinguir los siguientes tipos de barreras al empleo de las energías renovables a las redes eléctricas de las compañías distribuidoras de energía: 


\section{Barreras regulatorias y de política}

- Aún no hay suficientes políticas de fomento a las Energías Renovables que posibilite la inversión en pequeños sistemas de generación distribuida para usuarios comerciales y residenciales.

\section{Informativas}

- El nivel de conocimiento entre los consumidores y usuarios de las redes eléctricas sobre los beneficios del empleo de las ER en pequeños sistemas de generación; adicionalmente, existe un vacío de conocimiento entre los inversionistas sobre las oportunidades y los beneficios de ER.

- Debido al historial de altos precios de las tecnologías asociadas a las ER, lo que está cambiando en la actualidad, en el sector industrial las empresas que ofertan las tecnologías se enfrentan a dificultades al ofertar proyectos.

- Algunos agentes financieros también carecen de conocimiento sobre ER lo que dificulta el apalancamiento de proyectos que incluyen tecnología renovable.

\section{Institucionales y culturales}

- A nivel gubernamental se hacen necesarios organismos dedicados a promover las ER a nivel nacional e internacional.

- Ausencia de cultura sobre la conservación de la energía eléctrica que está asociada a la falta de confianza en la obtención de ahorros de energía resultantes del uso de ER.

\section{De mercados}

- Existencia de procesos de licitación desfavorables para las ER, especialmente en el sector público, donde las normas de contratación se guían por el precio más bajo y no la mejor relación costo/beneficios.

- Inversión Inicial elevada en la tecnología de ER, lo que se compensa si se comparan los costos nivelados para considerar tanto la inversión inicial como los costos de operación que en el caso de ER son menores.

- Empleo de pliegos tarifarios que no reflejan el costo real de la energía en algunos países debido a los subsidios indirectos a los derivados del petróleo.

\section{Técnicas}

- El usuario de las redes de distribución a nivel comercial y residencial no posee los conocimientos y la capacidad para desarrollar y poner en práctica proyectos de ER.

- Desarrollar tecnología asociada a la ER que sea relativamente fácil de instalar.

- Miedo a las interrupciones que podrían manifestarse si no se calculan adecuadamente los sistemas de generación distribuida.

- En la mayoría de nuestros países de américa Latina hay pocos proveedores de servicios de ER con la experiencia y capacidad para desarrollar e implementar proyectos llaves en mano.

\section{Financieras}

- Insolvencia financiera de muchos usuarios de la energía para recibir préstamos directos y aversión de muchos usuarios a asumir la deuda para proyectos de ER.

- La banca local tiene dificultades para evaluar la solidez técnica y económica de proyectos de ER en sistemas de generación distribuida.

- La falta de programas de financiamiento con tasas atractiva.

\section{CONCLUSIONES}

En este trabajo se analizaron las diferentes barreras que se presentan como un reto a mejorar para la inserción de las Energías Renovables (ER) en sistemas de generación distribuida a pequeña escala. Se abordó el marco normativo nicaragüense para valorar si facilita el empleo de ER para pequeños emprendimientos de generación de energía concluyéndose que aún hay que mejorar las normativas para hacer atractiva la inversión privada en esta actividad.

Además, haciendo uso de la teoría fundamentada se lograron detectar vía la indagación documental detectar una red de códigos que muestra las diferentes barreras a las energías renovables expresadas en los 
documentos revisados. Del mismo modo, se lograron identificar los diferentes tipos de barreras al empleo de las energías renovables a las redes eléctricas de las compañías distribuidoras de energía en base al estudio del caso nicaragüense.

\section{REFERENCIAS}

Cordech, M. (2009). ¿Renovables O Nuclear? ¿La economía política de la sostenibilidad energética? Ecología Política, (39), pp. 59-64.

Instituto Nicaragüense de la Energía, INE. (2021). Capacidad Instalada del sistema Eléctrico Nacional de Nicaragua. Recuperado de: https://www.ine.gob.ni/DGE/estadisticas/serieHistorica/capacidad-instaladaenergia-2010-2017-actabril18.pdf

Ortiz, D; Sabogal, J y Hurtado, E. (2012). Una revisión a la reglamentación e incentivos de las energías renovables en Colombia. rev.fac. cienc. econ., Volumen 20 (2), pp.55-67

Ovies, M. (2017). 14 cambios de paradigma a partir de la revolución de octubre de 1917: planificación, economía de la demanda y energías renovables. Ábaco, (91/92), pp. 163-172.

Elgamal, G y Demajorovic, J. (2020). Barreras y perspectivas para la generación de energía eléctrica por paneles fotovoltaicos solares en la matriz de energía brasileña. Revista de Gestão Ambiental $e$ Sustentabilidade (GeAS). Volumen 9 (1), pp.26. 26p. DOI: 10.5585/geas. v9i1.17157

Luna, I y Ramírez, Y. (2018). Energías renovables: una opción limpia para el ahorro de energía. Revista Ciencia Administrativa, pp.261-278.

Lara, P. (2015). Transición energética para un clima estable. Política Exterior, Volumen 29 (168). pp. 138148.

López, G; Isaac, A.; González, J; Cardona, H. (2014). Integración de energías renovables (solar fotovoltaica) en campus UPB Laureles-micro red inteligente. Revista Investigaciones Aplicadas, Volumen 8 (2) p157164.

Sanabria, S; Pedraza, Pablo y Hurtado, A. (2014). El emprendimiento como fuente de desarrollo y fortalecimiento de las capacidades endógenas para el aprovechamiento de las energías renovables. Revista EAN, (77), pp.19-167. 17p.

Turiel, A. El declive energético. (2012). Mientras tanto, (117), pp. 11-26.

\section{SEMBLANZA DE LOS AUTORES}

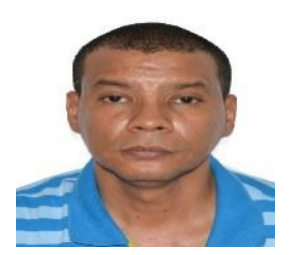

Napoleon V. Blanco Orozco: Doctor en Ciencias del Instituto Tecnológico de Costa Rica (ITCR) en 2015. Máster en Gestión de Proyectos en la UNI, 2009. Ingeniero eléctrico graduado en 1998 en la UNI. Es profesor de la Universidad Nacional de Ingenieria en el área de metodología de la investigación, máquinas eléctricas y la gestión de proyectos, Jefe del departamento y coordinador del grupo de investigación de ingeniería eléctrica en la UNI en la Facultad de Electrotecnia y Computación. 